\title{
Quantum mechanics of particles trapped in a Lamé circle or Lamé sphere shaped potential well
}

\author{
T. Isojärvi \\ Independent researcher, Vantaa, Finland
}

Received 30 November 2020; accepted 16 December 2020

\begin{abstract}
Ground state and 1st excited state energies and wave functions were calculated for systems of one or two electrons in a 2D and 3D potential well having a shape intermediate between a circle and a square or a sphere and a cube. One way to define such a potential well is with a step potential and a bounding surface of form $|x|^{q}+|y|^{q}+|z|^{q}=|r|^{q}$, which converts from a sphere to a cube when $q$ increases from 2 to infinity. This kind of geometrical object is called a Lamé surface. The calculations were done either with implicit finite difference time stepping in the direction of negative imaginary time axis or with quantum diffusion Monte Carlo. The results demonstrate how the volume and depth of the potential well affect the $E_{0}$ more than the shape parameter $q$ does. Functions of two and three parameters were found to be sufficient for fitting an empirical graph to the ground state energy data points as a function of well depth $V_{0}$ or exponent $q$. The ground state and first excited state energy of one particle in a potential well of this type appeared to be very closely approximated with an exponential function depending on $q$, when the well depth and area or volume was kept constant while changing the value of $q$. The model is potentially useful for describing quantum dots that deviate from simple geometric shapes, or for demonstrating methods of computational quantum mechanics to undergraduate students.
\end{abstract}

Keywords: Potential well; Lamé curve; superquadric; imaginary time; quantum dot; color center.

PACS: $03.65 . \mathrm{Ge}$

DOI: https://doi.org/10.31349/RevMexFis.67.206

\section{Introduction}

The model of an electron or some other particle confined inside a potential well of some shape, depth and volume is useful in many applications despite its simplicity. Examples of this include color centers in crystalline materials [1,2], quantum dots in nanotechnology $[3,4]$, pi electrons in conjugated organic molecules [5] and electron bubbles in liquid ammonia or liquid helium [6-8]. Despite the confinement of the electron being a result of complex multi-particle interactions, the particle-in-a-box model often produces at least qualitatively correct results.

\subsection{Potential wells with simple geometry}

Often a real-world object can be modeled with some exceptionally simple physical system. An electron in a potential well with the shape of an exact cube or a sphere, and the potential energy $V$ stepping abruptly at the well boundary is one example. In that situation, the potential energy of the electron is (spherical case)

$$
V(r)=V_{0} \Theta(r-\rho),
$$

or (cubic case)

$$
V(x, y, z)=V_{0} \Theta(\max (|x|,|y|,|z|)-L / 2) .
$$

Here the $V_{0}$ is the depth of the well. $\Theta$ is the Heaviside theta function,

$$
\Theta(x)=\left\{\begin{array}{ll}
0, & \text { when } x<0, \\
1, & \text { when } x \geq 0
\end{array},\right.
$$

$\rho$ is the radius of the circular or spherical potential well and $L$ is the side length of the cubic potential well. The variable $r$ is just the distance from the origin in 2D or 3D space: $r=\sqrt{x^{2}+y^{2}}$ or $r=\sqrt{x^{2}+y^{2}+z^{2}}$. The function $V$ appears in the time-independent Schrödinger equation, from which the energy eigenvalues and eigenfunctions are calculated

$$
-\frac{\hbar^{2}}{2 m} \nabla^{2} \psi(\mathbf{x})+V(\mathbf{x}) \psi(\mathbf{x})=E \psi(\mathbf{x}) .
$$

Here $\mathbf{x}$ is a two- or three-dimensional vector.

\subsection{Lamé circles and spheres}

In real-world situations it can not always be assumed that the electron is confined to an exactly spherical or cubic space. The choice $V_{0}=\infty$ is another crude approximation. A logical improvement to the model would be to define 2D curves and 3D surfaces with a shape between a square and a circle or cube and a sphere. One geometrical object of this type is the Lamé circle (or supercircle)

$$
\frac{|x|^{q}}{a^{q}}+\frac{|y|^{q}}{b^{q}}=r^{q},
$$

and the Lamé sphere (or supersphere)

$$
\frac{|x|^{q}}{a^{q}}+\frac{|y|^{q}}{b^{q}}+\frac{|z|^{q}}{c^{q}}=r^{q} .
$$

Yet another name for these curves and surfaces is superquadrics. The objects described by these equations, with $a=b=c=1$ (as in all cases studied in this article), become 


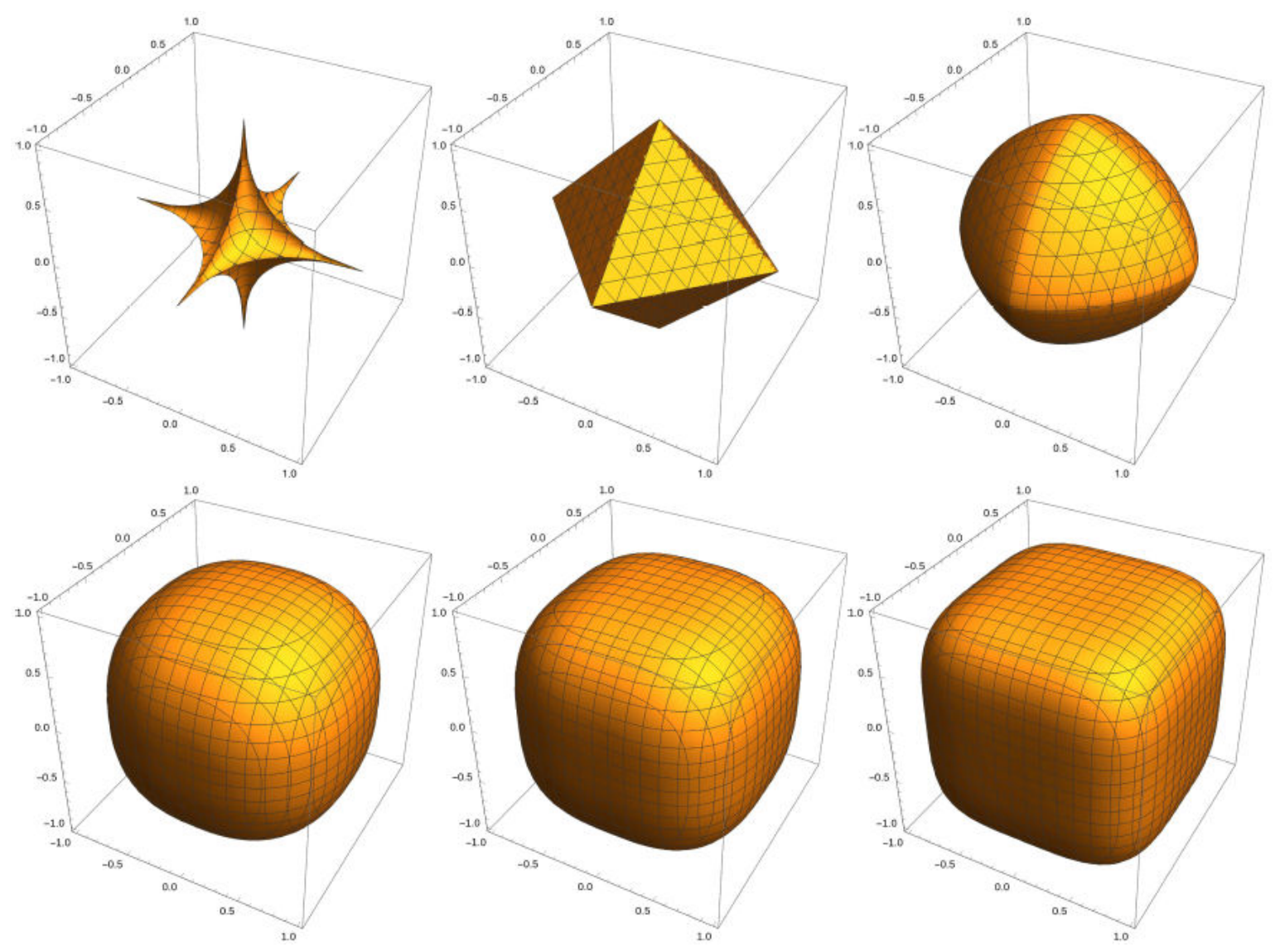

FIGURE 1. Wolfram Mathematica 11.2.0 plots of the surface defined by $|x|^{q}+|y|^{q}+|z|^{q}=r^{q}$ for values $q=0.5$, $q=1.0 q=1.5$, $q=2.5, q=3$ and $q=4$ from left to right and up to down. The parameter $r$ has been set to value 1 .

a circle or sphere when $q=2$ and a square or a cube when $q \rightarrow \infty$. The parameter $r>0$ is the radius of the circle or sphere that these shapes become when $q \rightarrow 2$. As a general result, an $n$-dimensional solid of this shape has volume [9]

$$
\operatorname{Vol}(r, n, q)=(2 r)^{n} \frac{\Gamma(1+1 / q)^{n}}{\Gamma(1+n / q)}
$$

where $\Gamma$ is the gamma function. To produce a set of surfaces described by (6), having different values of $q$ but same constant enclosed volume $\mathcal{V}$, the parameter $r$ in Eq. (6) has to be made a function of $q$ :

$$
r:=r(q)=\sqrt[3]{\frac{\mathcal{V}}{\operatorname{Vol}(1,3, q)}},
$$

or the equivalent for a 2D Lamé circle.

3D surfaces of shapes given by Eq. (6) with $a=b=c=$ 1 are shown in Fig. 1.

The system with a potential well bounded by a Lamé curve has been studied earlier by Bera et al. [10], but only for the 2D case and with infinite $V_{0}$. In this article, the results will be extended to the equivalent $3 \mathrm{D}$ systems with several finite values of $V_{0}$ allowed.

A similar method of analytical geometry exists for converting a sphere continuously into a Platonic solid (regular octahedron, dodecahedron or icosahedron instead of a cube) $[11,12]$, and it can be expected that the energy eigenvalues of an electron in that kind of potential well approach the spherical case when more faces are added to the polyhedron.

\subsection{Experimental methods}

A practical system that can be modelled as one or more electrons in a 1 to 3-dimensional potential well is the quantum dot (QD), which is a nanometer-scale semiconductor particle having an absorption/emission spectrum similar to a system of electrons confined in a potential well of equivalent size. An electron in a large QD has a lower ground state energy than one in a small QD, just like an electron in a short versus long 1D square well. One complication in this theory of QDs is the -possibly position-dependent- difference of electron and hole effective masses to their normal value or the value in bulk semiconductor [13]. This kind of nanoparticles can be produced by chemical or mechanical means such as molecular beam epitaxy or chemical vapor deposition [14], and their shape can be probed by methods such as measuring their spin and parity oscillations in a magnetic field [15]. Information about the effect of shape changes, as in the model systems of this article, on the electronic spectrum of the system can also be utilized for quantum dot shape determination. Conversely, 
this also means that QDs with desired spectral properties can be produced by tuning their shape in an appropriate way.

\subsection{Computational approach}

The methods applied in this article are numerical, with ground state and first excited state energies obtained with quantum diffusion Monte Carlo (QDMC) or Implicit finite differencing (IFD) on imaginary time axis. The system is a 2D or 3D potential well with boundary described by Eqs. (5) and (6). The parameters $a, b$ and $c$ all have value 1 , so the well is not oblong in any particular direction. The system contains one or two confined electrons. A suitable nonlinear fitting function is sought for the data points of ground state energy $E_{0}$ as a function of $q$ and $V_{0}$. This is done to make it easier to estimate the $E_{0}$ for other values of $q, V_{0}$ by interpolation. With spectroscopic data and these results, it may be possible to estimate whether an electron-trapping cavity in a real material is more close to sphere or cube in shape.

Both the QDMC and IFD methods for finding the ground state are based on the time evolution of an initial state $|\Psi(0)\rangle=c_{0}\left|\psi_{0}\right\rangle+c_{1}\left|\psi_{1}\right\rangle+c_{2}\left|\psi_{2}\right\rangle \ldots$ written as a linear combination of the eigenstates of $\hat{H},\left|\psi_{n}\right\rangle$ :

$$
\begin{aligned}
|\Psi(t)\rangle & =c_{0} e^{-i E_{0} t / \hbar}\left|\psi_{0}\right\rangle \\
& +c_{1} e^{-i E_{1} t / \hbar}\left|\psi_{1}\right\rangle+c_{2} e^{-i E_{2} t / \hbar}\left|\psi_{2}\right\rangle \ldots
\end{aligned}
$$

Here it is assumed that $\hat{H}$ has no explicit time dependence in Schrödinger picture. Defining an imaginary time coordinate with equation $t=-i \tau$, the time evolution in terms of $\tau$ is

$$
\begin{aligned}
|\Psi(\tau)\rangle & =c_{0} e^{-E_{0} \tau / \hbar}\left|\psi_{0}\right\rangle \\
& +c_{1} e^{-E_{1} \tau / \hbar}\left|\psi_{1}\right\rangle+c_{2} e^{-E_{2} \tau / \hbar}\left|\psi_{2}\right\rangle \ldots
\end{aligned}
$$

Provided that the $E_{n}$ form an increasing sequence of positive numbers, the state vector $|\Psi(\tau)\rangle$ of (10) approaches something proportional to $\left|\psi_{0}\right\rangle$ when $\tau \rightarrow$ $\infty$. This allows the numerical calculation of the ground state wave function $\psi_{0}(\mathbf{x})=\left\langle\mathbf{x} \mid \psi_{0}\right\rangle$. The eigenvalue $E_{0}$ can be obtained by following the expectation value $\langle\Psi(\tau)|\hat{H}| \Psi(\tau)\rangle /\langle\Psi(\tau) \mid \Psi(\tau)\rangle$, or the value of $\hat{H} \Psi(\mathbf{x}, \tau) / \Psi(\mathbf{x}, \tau)$ at some constant position $\mathbf{x}$, during the simulation.

The difference between the QDMC and IFD methods is that in QDMC the diffusive imaginary-time dynamics is modelled as a system of random walkers with a source term allowing creation and destruction of them. The IFD method calculates actual wave functions that do not have to be real and positive valued.

\section{Numerical methods}

The diffusion Monte Carlo calculation was done with a C++ source code described in [16] and appropriately modified to fit this particular problem. The code utilizes atomic-like units with Planck constant and electron mass set to $\hbar=m_{e}=$ 1. In the two-electron calculations, the Coulomb constant $\left(4 \pi \epsilon_{0}\right)^{-1}$ and the elementary charge $e$ also have value 1 . The implicit finite differencing with an imaginary time coordinate was done with a script written in $\mathrm{R}$ language. There the unit system is also one where both length and energy are dimensionless.

\subsection{Transcendental equation and shooting method for $q=2$ and $q=\infty$}

\subsubsection{Solution with non-algebraic equations for $q=2$ and $q=\infty$}

The calculation of the energy eigenvalues for a finite square, cubic or spherical potential well is described in most basic QM textbooks such as [17]. The method is based on the graphical solution of a non-algebraic (transcendental) equation. For a 1D square well with potential energy

$$
V(x)=\left\{\begin{array}{cl}
-V_{0}, & \text { when }|x|<\frac{L}{2} \\
0, & \text { when }|x| \geq \frac{L}{2}
\end{array},\right.
$$

the equation is

$$
\alpha \tan \left(\frac{\alpha L}{2}\right)=\beta
$$

for the even states such as the ground state, and

$$
\alpha \cot \left(\frac{\alpha L}{2}\right)=-\beta
$$

for the odd states like the 1 st excited state. The quantities $\alpha$ and $\beta$ are defined as

$$
\begin{aligned}
& \alpha=\left(\frac{2 m}{\hbar^{2}}\left(V_{0}+E\right)\right)^{1 / 2} \text { and } \\
& \beta=\left(-\frac{2 m}{\hbar^{2}} E\right)^{1 / 2} .
\end{aligned}
$$

These equations can be solved graphically after a straightforward conversion to units where $\hbar=m=1$ and to the convention of $V$ being 0 inside the well and $V_{0}$ outside it. The ground state energy of a square $2 \mathrm{D}$ or cubic $3 \mathrm{D}$ potential well is just 2 or 3 times the $E_{0}$ calculated for this 1-dimensional system. Also, the first excited state energy $E_{1}$ of a $2 \mathrm{D}$ squareshaped potential well with side length $L$ is the sum of $E_{0}$ and $E_{1}$ of the 1D potential well of length $L$.

The equation for solving energy eigenvalues of a 3D spherical potential well,

$$
V(r)=\left\{\begin{array}{cc}
-V_{0}, & \text { when } r<a \\
0, & \text { when } r \geq a
\end{array},\right.
$$

is of form

$$
K \cot K a=-\lambda,
$$

with

$$
K=\left[\frac{2 m}{\hbar^{2}}\left(E+V_{0}\right)\right]^{1 / 2} \text { and } \lambda=\left(-\frac{2 m E}{\hbar^{2}}\right)^{1 / 2} \text {. }
$$


However, Eq. (16) is only for rotation invariant eigenstates with zero angular momentum, of which the ground state is one example. For numerical work, these quantities have to be made dimensionless and shifted to make the interior of the potential well have $V=0$ as defined in this article.

\subsubsection{Shooting method}

For the 2D circular potential well, the corresponding equation contains special functions, and the result can more easily be obtained with the shooting method for the $L=0$ eigenstates. The Schrödinger equation can be cast into polar coordinates

$$
\begin{array}{r}
-\frac{\hbar^{2}}{2 m}\left(\frac{\partial^{2}}{\partial r^{2}}+\frac{1}{r} \frac{\partial}{\partial r}+\frac{1}{r^{2}} \frac{\partial^{2}}{\partial \theta^{2}}\right) \psi(r, \theta) \\
+V_{0} \Theta(r-\rho) \psi(r, \theta)=E \psi(r, \theta),
\end{array}
$$

which simplifies for the rotation invariant ground state

$$
\begin{aligned}
& -\frac{\hbar^{2}}{2 m}\left(\frac{\partial^{2}}{\partial r^{2}}+\frac{1}{r} \frac{\partial}{\partial r}\right) \psi(r) \\
& \quad+V_{0} \Theta(r-\rho) \psi(r)=E \psi(r) .
\end{aligned}
$$

The equation above can be solved numerically by changing to dimensionless variables and choosing a trial value of $E$ and a small number $\epsilon \ll 1$. Then it is integrated by finite difference starting from $r=\epsilon$ (not $r=0$, to avoid problems with the singular multiplier $1 / r$ in the polar Laplacian) with initial conditions $\psi(\epsilon)=1$ and $\psi^{\prime}(\epsilon)=0$. The value of $E$ is adjusted until the radial wave function doesn't blow up to negative or positive infinity near the potential step $r=\rho$. The smallest value of $E$ with this property can be taken as an approximation for $E_{0}$. The same procedure can also be applied for finding the energies $E_{n}$ of other rotation invariant eigenfunctions of the Hamiltonian, but the first excited state with energy $E_{1}$ is not one of those.

\subsection{Quantum DMC for one particle in $2 D$ and 3D}

The diffusion Monte Carlo results for both 2D and 3D were calculated with values of $2 \leq q \leq 20$ and $50 \leq V_{0} \leq 20000$. The result for each combination of $q$ and $V_{0}$ was calculated 4 times and averaged to one data point. The time step length in both $2 \mathrm{D}$ and $3 \mathrm{D}$ simulations was $\Delta t=10^{-5}$, the energy update parameter was $\alpha=40$ and the maximum allowed number of random walkers was $10^{5}(2 \mathrm{D})$ or $9 \times 10^{5}(3 \mathrm{D})$. Simulated time length was 20 units, and 1/4 from the beginning of the energy output file was discarded before averaging the remaining energy values to an estimate for $E_{0}$.

The results were plotted as graphs where either $V_{0}$ or $q$ was kept constant and the other varied. Particle mass was set to 1 in all calculations. The area of a 2D potential well was kept constant at $A=4$ when changing the value of the exponent $q$, and the volume of the 3D potential well was kept at $\mathcal{V}=8$. This was done by defining $r=r(q)$ as in (8).

Calculations for well depths of 600, 2000 and 20000 were also done similarly for a $3 \mathrm{D}$ well of volume $\mathcal{V}=2.0$ to see the effect of volume change on the ground state energy. In the $\mathcal{V}=2.0$ calculation, the time step was $\Delta t=5 \times 10^{-6}$, the maximum number of walkers was 90000 , length of simulation was 9.0 time units and the energy update parameter $\alpha$ had value 100.0.

In preliminary calculations, it was seen that an exponential function of type

$$
E_{0}(q)=A_{0}-\left(A_{0}-A_{1}\right) e^{A_{2}(q-2)}
$$

fits well in the data points of $E_{0}$ as a function of $q$, and a rational square root function

$$
E_{0}\left(V_{0}\right)=A_{0}-\frac{A_{1}}{\sqrt{V_{0}}}
$$

to the data points of $E_{0}$ as a function of $V_{0}$. Making Eq. (20) more complex by converting it to a sum of two exponentials did not add any more accuracy in the fit. Both kind of graphs (as a function of $q$ or $V_{0}$ ) can be used for estimating the ground state energy for intermediate values of $q$ and $V_{0}$ by interpolation. There is no obvious theoretical reason for the $E_{0}\left(V_{0}\right)$ and $E_{0}(q)$ to be close to these functions; the reason for calculating the nonlinear fit is just to predict the behavior of the functions between calculated data points.

The results were plotted and the iterative nonlinear fits calculated with the Linux application Grace-5.1.25. The obtained values of fitting parameters $A_{0}, A_{1}$ and $A_{2}$ were recorded for all cases. Another way to do the curve fit is to fix $A_{0}$ and $A_{1}$ to values predicted by a transcendental equation or with the shooting method, and leave only $A_{2}$ free.

\subsection{Quantum DMC for two particles in 2D}

\subsubsection{Basic parameters}

The problem of two electrons in the same potential well was numerically investigated only in 2D to limit the computation time. Instead of having only $q$ and $V_{0}$ as parameters, the area of the potential well was also varied to see its effect on $E_{0}$. The maximum number of random walkers in these simulations was 30000 and the time step was $3 \times 10^{-6}$. The energy update parameter $\alpha$ needed to have higher value, up to 120 , than in the 1-particle version to give better accuracy.

The value of $q$ in these results was either 2,8 or $\infty$. The well depth was $V_{0}=200$ or 20000 . The size of the potential well was parametrized with a variable $s$, with the area of the potential well being $\pi$ when $s=1$ and $\pi s^{2}$ for other values of $s$. In other words, $s$ is a multiplier of all linear dimensions of the system. The parameter $s$ was varied from $s=1$ to 4 in steps of 0.5 . In addition to the ground state energy $E_{0}$, the mean electron-electron distance $r_{12}$ was extracted from the output wave function. 


\subsubsection{Electron-electron interaction energy}

The position representation Hamiltonian of two interacting electrons confined in a supercircular potential well is

$$
\hat{H}=-\frac{\hbar^{2}}{2 m_{e}}\left(\nabla_{1}^{2}+\nabla_{2}^{2}\right)+\hat{V}_{\mathrm{conf}}+\frac{e^{2}}{4 \pi \epsilon_{0} \hat{r}_{12}},
$$

where the $\nabla_{1}^{2}$ and $\nabla_{2}^{2}$ are two-dimensional Laplacians acting on the coordinates of the 1 st and 2 nd electron. The op-

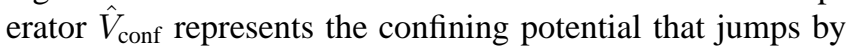
amount $V_{0}$ when an electron crosses the boundary of the supercircle. The term proportional to $\left(\hat{r}_{12}\right)^{-1}$ is the electronelectron interaction energy, and becomes just $\left(\hat{r}_{12}\right)^{-1}$ in the dimensionless calculations with atomic units. If $x_{1}, y_{1}$ are the coordinates of first electron and $x_{2}, y_{2}$ are those of second electron, the value used for distance $r_{12}$ is $r_{12}=$ $\sqrt{\left(x_{1}-x_{2}\right)^{2}+\left(y_{1}-y_{2}\right)^{2}+\epsilon}$. The $\epsilon$ here is some small positive constant that prevents division by zero in the calculations. In this work, its value was $\epsilon=10^{-3}$ and one simulation was done with $\epsilon=10^{-5}$ to verify that this doesn't affect the values of $E_{0}$ significantly.

A system of only two electrons does not require any special care to antisymmetrize the ground state wave function, because the two electrons can have opposite spins.

\subsection{Implicit finite-differencing for one particle in $2 \mathrm{D}$}

\subsubsection{Description}

In the simplest possible case of a 1D quantum system, the time-dependent Schrödinger equation is made discrete by replacing the wave function $\Psi(x, t)$ with an array $\Psi_{j}^{k}$. The $j$ is a position index and $k$ a time index. If the coordinate system is defined so that the interesting part of the system is on the spatial interval $x \in\left[0, x_{\max }\right]$ and the temporal interval $t \in\left[0, t_{\max }\right]$, then the equivalence is like $\Psi_{j}^{k} \leftrightarrow \Psi(j \Delta x / M, k \Delta t / N)$. Here the $M$ is the maximum value of the position index, $N$ the maximum value of time index, $\Delta x=x_{\max } / M$ and $\Delta t=t_{\max } / N$.

As described in [19], the Crank-Nicolson method is a way to turn the discrete version of the Schrödinger equation into an implicit scheme that preserves unitarity. This is done by approximating the evolution operator $\hat{U}_{\Delta t}$ with the Cayley's form

$$
\hat{U}_{\Delta t}=e^{-i \hat{H} \Delta t} \approx \frac{1-\frac{1}{2} i \hat{H} \Delta t}{1+\frac{1}{2} i \hat{H} \Delta t}
$$

where the units have been chosen so that $\hbar=1$. Noting that $\Psi_{j}^{k+1}=\hat{U}_{\Delta t} \Psi_{j}^{k}$, this approximation leads to the result

$$
\left(1+\frac{1}{2} i \hat{H} \Delta t\right) \Psi_{j}^{k+1}=\left(1-\frac{1}{2} i \hat{H} \Delta t\right) \Psi_{j}^{k}
$$

If the operator $\hat{H}$ is simply a sum of a kinetic and poten- tial energy term as in (4), the equation is

$$
\begin{aligned}
\Psi_{j}^{k+1} & -\frac{i}{4} \Delta t\left(\frac{\Psi_{j+1}^{k+1}-2 \Psi_{j}^{k+1}+i \Delta t V_{j}+\Psi_{j-1}^{k+1}}{[\Delta x]^{2}}\right)=\Psi_{j}^{k} \\
& +\frac{i}{4} \Delta t\left(\frac{\Psi_{j+1}^{k}-2 \Psi_{j}^{k}+\Psi_{j-1}^{k}}{[\Delta x]^{2}}\right)-i \Delta t V_{j}
\end{aligned}
$$

With $V_{j}$ the array of position-dependent potential energy values. This is a tridiagonal system of linear equations for solving the array $\Psi_{j}^{k+1}$ when $\Psi_{j}^{k}$ is known. The Eq. (25) can be colloquially described as a statement that "the result of propagating the state $\Psi_{j}^{k}$ forward in time by half a timestep is the same as that of propagating $\Psi_{j}^{k+1}$ backwards by half a timestep".

\subsection{2. $2 D$ systems}

In the 2D case, the discrete object replacing the wave function $\Psi(x, y, t)$ is a three-index array $\Psi_{j, k}^{l}$, with $j, k$ position indices and $l$ a time index. In that situation, the equation corresponding to $(25)$ is

$$
\begin{aligned}
\Psi_{j, k}^{l+1} & -\frac{i}{4} \Delta t\left(\frac{\Psi_{j+1, k}^{l+1}-2 \Psi_{j, k}^{l+1}+\Psi_{j-1, k}^{l+1}}{[\Delta x]^{2}}\right. \\
& \left.+\frac{\Psi_{j, k+1}^{l+1}-2 \Psi_{j, k}^{l+1}+\Psi_{j, k-1}^{l+1}}{[\Delta x]^{2}}\right)+i \Delta t V_{j, k}=\Psi_{j, k}^{l} \\
& +\frac{i}{4} \Delta t\left(\frac{\Psi_{j+1, k}^{l}-2 \Psi_{j, k}^{l}+\Psi_{j-1, k}^{l}}{[\Delta x]^{2}}\right. \\
& \left.+\frac{\Psi_{j, k+1}^{l}-2 \Psi_{j, k}^{l}+\Psi_{j, k-1}^{l}}{[\Delta x]^{2}}\right)-i \Delta t V_{j, k}
\end{aligned}
$$

Here it is assumed that the spatial step size is the same $\Delta x$ to both $x$ and $y$ directions. However, this equation cannot be written as a tridiagonal system anymore, and this makes the computational cost of the problem increase very rapidly with increasing size of the discrete $x-y$ grid. More specifically, the number of arithmetic operations needed for solving a tridiagonal system of $n$ equations and $n$ unknowns scales as $\mathcal{O}(n)$ while that for a general linear system scales as $\mathcal{O}\left(n^{3}\right)$.

\subsubsection{Operator splitting}

One way to circumvent this problem for large discrete systems is to approximately divide every time step to three parts. In the first part, the $\mathrm{x}$-direction kinetic energy is accounted for by solving

$$
\begin{gathered}
\Psi_{j, k}^{l+1 / 3}-\frac{i}{4} \Delta t\left(\frac{\Psi_{j+1, k}^{l+1 / 3}-2 \Psi_{j, k}^{l+1 / 3}+\Psi_{j-1, k}^{l+1 / 3}}{[\Delta x]^{2}}\right) \\
=\Psi_{j, k}^{l}+\frac{i}{4} \Delta t\left(\frac{\Psi_{j+1, k}^{l}-2 \Psi_{j, k}^{l}+\Psi_{j-1, k}^{l}}{[\Delta x]^{2}}\right) .
\end{gathered}
$$


The second equation is the same for y-kinetic energy:

$$
\begin{aligned}
& \Psi_{j, k}^{l+2 / 3}-\frac{i}{4} \Delta t\left(\frac{\Psi_{j, k+1}^{l+2 / 3}-2 \Psi_{j, k}^{l+2 / 3}+\Psi_{j, k-1}^{l+2 / 3}}{[\Delta x]^{2}}\right) \\
& =\Psi_{j, k}^{l}+\frac{i}{4} \Delta t\left(\frac{\Psi_{j, k+1}^{l+1 / 3}-2 \Psi_{j, k}^{l+1 / 3}+\Psi_{j, k-1}^{l+1 / 3}}{[\Delta x]^{2}}\right),
\end{aligned}
$$

and the third equation is just a multiplication with a complex phase factor

$$
\Psi_{j, k}^{l+1}=\exp \left(-i \Delta t V_{j, k}\right) \Psi_{j, k}^{l+2 / 3} .
$$

The justification of doing this is that for $\Delta t \ll 1$, the evolution operator $\hat{U}_{\Delta t}$ can be approximately divided to parts

$$
\begin{aligned}
\hat{U}_{\Delta t} & =e^{-i \hat{H} \Delta t}=\exp \left(-i \Delta t\left(\hat{K}_{x}+\hat{K}_{y}+\hat{V}\right)\right) \\
& \approx e^{-i \hat{K}_{x} \Delta t} e^{-i \hat{K}_{y} \Delta t} e^{-i \hat{V} \Delta t}
\end{aligned}
$$

despite $\hat{K}_{x}, \hat{K}_{y}$ and $\hat{V}$ not necessarily commuting. This results from the Trotter product formula [18].

Both (27) and (28) can be written as tridiagonal matrixvector equations, and the multiplication (29) is a trivial calculation. The time step $\Delta t$ has to be smaller than when solving the dynamics from (26), but this operator splitting method still produces a significant increase in computational speed for large grids of points $(j, k)$. This operator splitting method and its modifications have been used, for instance, in [20] in the context of wavepacket dynamics and in [18] to factorize operators in quantum statistical mechanics.

\subsubsection{Simulation parameters}

In the actual simulations, the domain in xy-plane was a square with opposing corners at $(0,0)$ and $(3.4,3.4)$. This domain is large enough to contain any potential well of area 4.0 and shape given by (5) with $2 \leq q<\infty$. Enough grid points are also left outside the well for the wave function to practically reach zero before the boundaries of the domain (given that the depth of the well is $V_{0} \geq 2000$ ). The center of the potential well is at $(x, y)=(1.7,1.7)$, and the spatial discretization contains $850 \times 850$ points. The function "Solve.tridiag" from the limSolve package was used for solving the linear systems in $\mathrm{R}$.

The time step was $\Delta t=10^{-4}$ in all cases, and the simulation length on imaginary time axis had to be $t_{\max }>1.5$ for good convergence. Once the ground state quantities $E_{0}$ and $\left\langle x \mid \psi_{0}\right\rangle$ were calculated by this scheme, the 1 st excited state was obtained in the same way with the ground state component removed from $\Psi$ on each time step with projection: $|\Psi\rangle \mapsto|\Psi\rangle-\left\langle\psi_{0} \mid \Psi\right\rangle\left|\psi_{0}\right\rangle$.

The initial state $\Psi_{i, j}^{0}$ was built from Gaussian functions in a way that ensures that it wasn't either even or odd to any Cartesian direction w.r.t. the center of the potential well. This was to make sure that the initial condition $\left|\psi\left(t_{0}\right)\right\rangle$ isn't orthogonal to either $\left|\psi_{0}\right\rangle$ or $\left|\psi_{1}\right\rangle$.

The eigenvalues $E_{0}$ and $E_{1}$ were calculated for $V_{0}=$ 2000 and $V_{0}=20000$, and for all values of $q$ from the set $\{2,4,6,8,10,15\}$. After all data points $E_{0}\left(V_{0}, q\right)$ and $E_{1}\left(V_{0}, q\right)$ were obtained, the function (21) was fitted to all result sets.

In addition to that mentioned above, the 2 nd excited state energy $E_{2}$ was calculated for the $q=2$ case with both $V_{0}=2000$ and $V_{0}=20000$. This was done by doing another simulation where the wave function was orthogonalized with both $\left|\psi_{0}\right\rangle$ and $\left|\psi_{1}\right\rangle$ on each time step. The reason for calculating this was that the first excited state $\left|\psi_{1}\right\rangle$ and its energy eigenvalue $E_{1}$ can't be obtained by the shooting method, because it is not rotation invariant. Comparison of the $E_{2}$ from IFD integration to the value solved with Eq. (19) is one way to verify the correctness of the IFD method applied here.

\section{Results}

\subsection{Ground state energy of $q=2$ and $q=\infty$ potential wells}

The results for the $E_{0}$ of square/cubic systems with side length 2, obtained from the graphical solution of Eq. (12), are on the left side of Table I.

The equivalent results for 2D circular or 3D spherical potential wells, with area 4 or volume 8 are shown on the right side of Table I. The former has been obtained with the shooting method and the latter with Eq. (16).

\subsection{QDMC results for one particle in $2 D$ or $3 D$}

For both $2 \mathrm{D}$ and $3 \mathrm{D}$, and for every value of $V_{0}$ from 50 to 20000, 12 ground state energy data points from $q=2$ to $q=20$ were obtained in the QDMC calculation. To each point set of different $V_{0}$, the exponential function of (20) was fitted to the $E_{0}(q)$ data. This was done both with all parameters $A_{0}, A_{1}$ and $A_{2}$ free, or with $A_{0}$ and $A_{1}$ fixed to the limiting values of $E_{0}(q=2)$ and $E_{0}(q \rightarrow \infty)$ for that $V_{0}$

TABLE I. The ground state energy, $E_{0}$ of a particle of mass 1 in a $2 \mathrm{D}$ or $3 \mathrm{D}$ potential well with $q=\infty$ (left table) or $q=2$ (right table), area 4.0 and height of potential barrier $V_{0}$.

\begin{tabular}{ccccc}
\hline$V_{0}$ & $E_{0}$ (square) & $E_{0}$ (cube) & $E_{0}$ (circle) & $E_{0}$ (sphere) \\
\hline 50 & 2.0380 & 3.0570 & 1.9140 & 2.7146 \\
100 & 2.1518 & 3.2277 & 2.0102 & 2.8679 \\
200 & 2.2378 & 3.3567 & 2.0821 & 2.9617 \\
600 & 2.3308 & 3.4962 & 2.1591 & 3.0616 \\
1200 & 2.3696 & 3.5544 & 2.1911 & 3.1028 \\
2000 & 2.3912 & 3.5868 & 2.2087 & 3.1256 \\
10000 & 2.4328 & 3.6492 & 2.2428 & 3.1696 \\
20000 & 2.4430 & 3.6645 & 2.2511 & 3.1801 \\
\hline
\end{tabular}



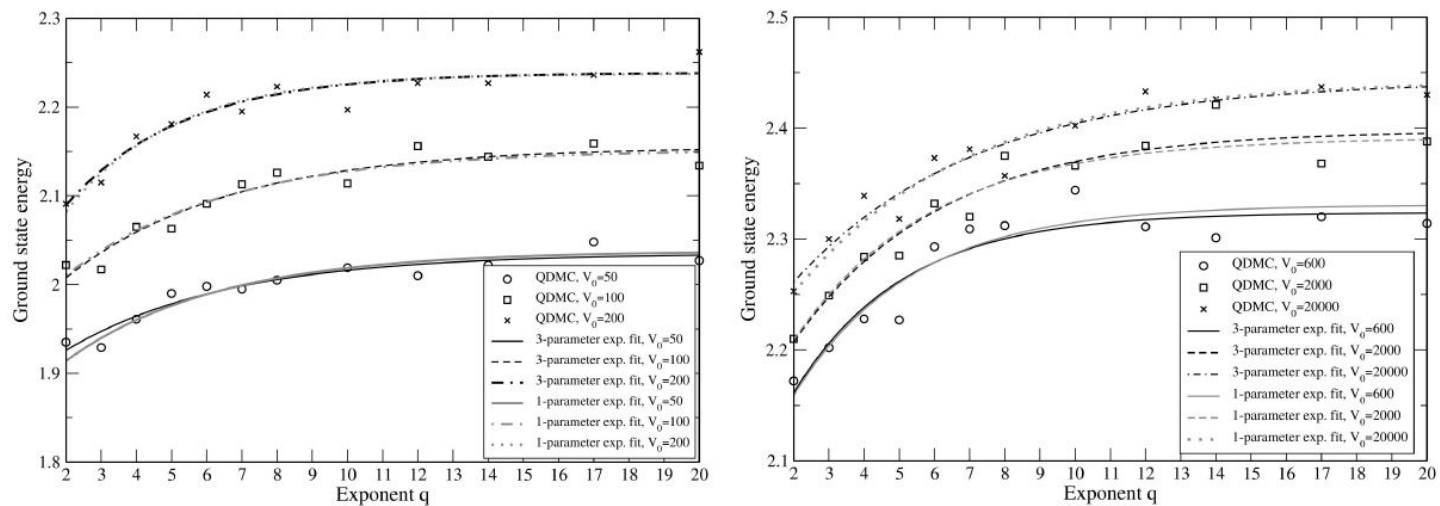

FIGURE 2. QDMC ground state energies and 1- or 3-parameter exponential curve fits (20) for one electron in a 2D potential well of shape (5) and area 4.0, and with different values of exponent $q$. The units have been chosen so that $\hbar=m_{e}=1$ and energy is dimensionless.
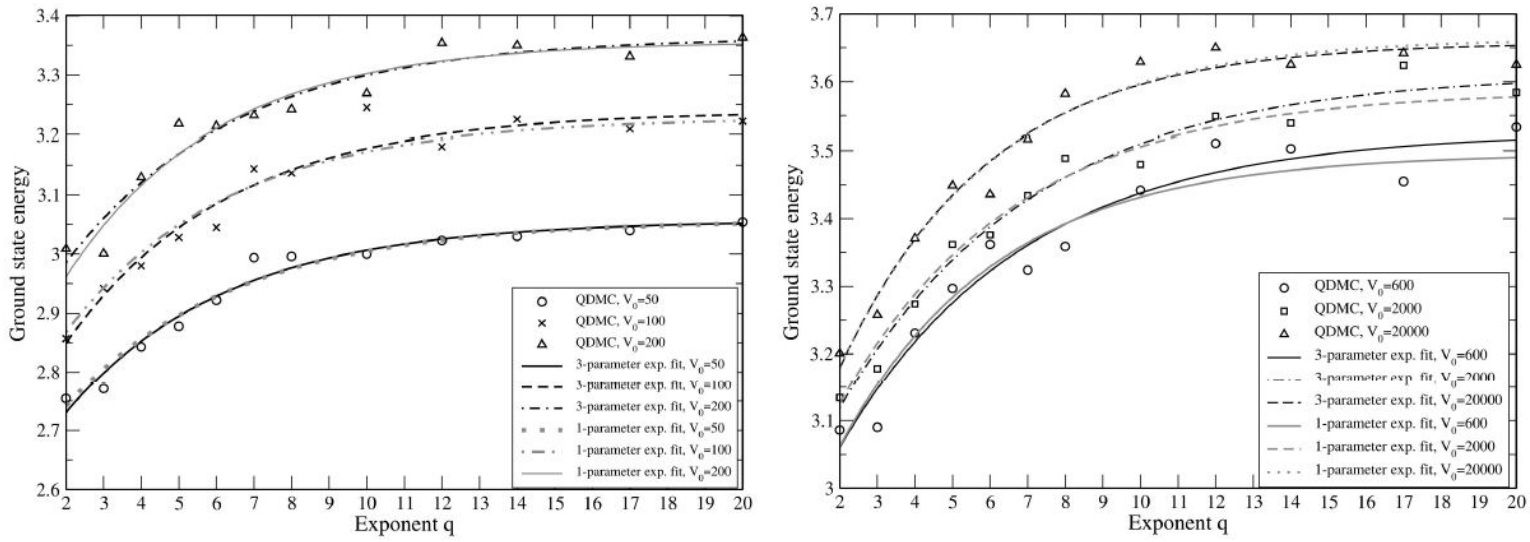

FIGURE 3. QDMC ground state energies and 1- or 3-parameter exponential curve fits (20) for one electron in a 3D potential well of shape (6) and volume 8.0, and with different values of exponent $q$. The dimensionless units are again $\hbar=m_{e}=1$.

(Table I) and only $A_{2}$ left free. Figures 2 and 3 show both the $E_{0}$ data points and the 1- or 3-parameter curve fits to the data with a potential well of area $A=4.0$ or volume $\mathcal{V}=8.0$.

The similarity of the 1-parameter (gray lines) and 3parameter (black lines) exponential fits in Figs. 2 and 3 reflects how accurately the $E_{0}$ values calculated with QDMC agree with those calculated by different means for the $q=2$ and $q=\infty$ limiting cases (Table I).

Main important features in the data of Fig. 2 and 3 include that the $E_{0}$ grows monotonically with increasing $q$ in all cases, and the difference in $E_{0}$ between $q=2$ and $q=\infty$ is in the range of $5-15 \%$. Most of the change in ground state energy in all data sets has already taken place when $q$ has increased to value 8 from the original 2. The nonlinear fit parameter $A_{2}$ was between -0.30 and -0.20 for almost all data sets.

Figure 4 shows the additional results $E_{0}(q)$ for the three cases of a 3D potential well of smaller volume 2.0. The graphs look similar to those for larger potential wells, except for the $E_{0}$ values being higher. The curve fit parameters of Figs. 2 to 6 are also included as tables of numerical data in the Appendix.

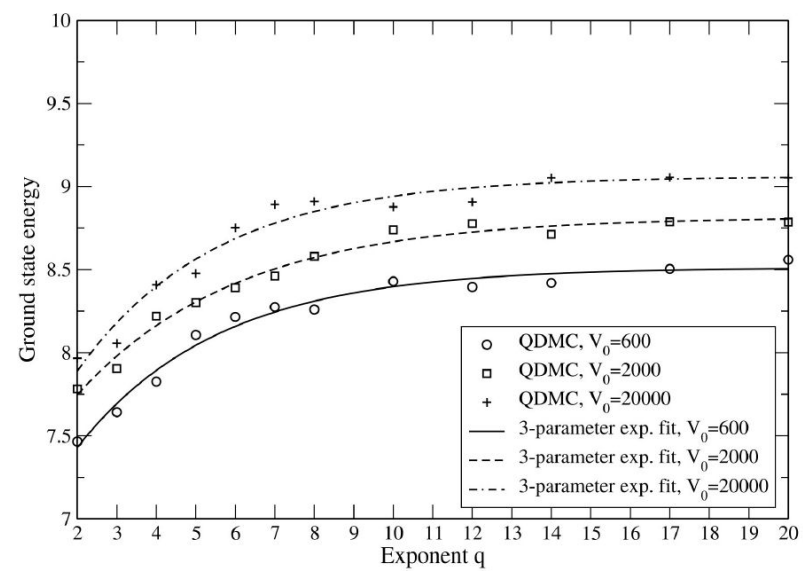

FIGURE 4. QDMC $E_{0}$ values and 3-parameter exponential curve fits (20) for one electron in a 3D potential well of shape (6) and volume 2.0, and with exponent $q$ as the independent variable.

It is also possible to present the data as graphs with $q$ and $\mathcal{V}$ kept constant and $V_{0}$ varied. The square root type function (21) was fitted iteratively to $E_{0}\left(V_{0}\right)$ data sets corresponding to different values of $q$. The parameters $A_{0}$ and $A_{1}$ in Eq. (21) were free, and not pre-fixed, in each case. Figures 5 and 6 

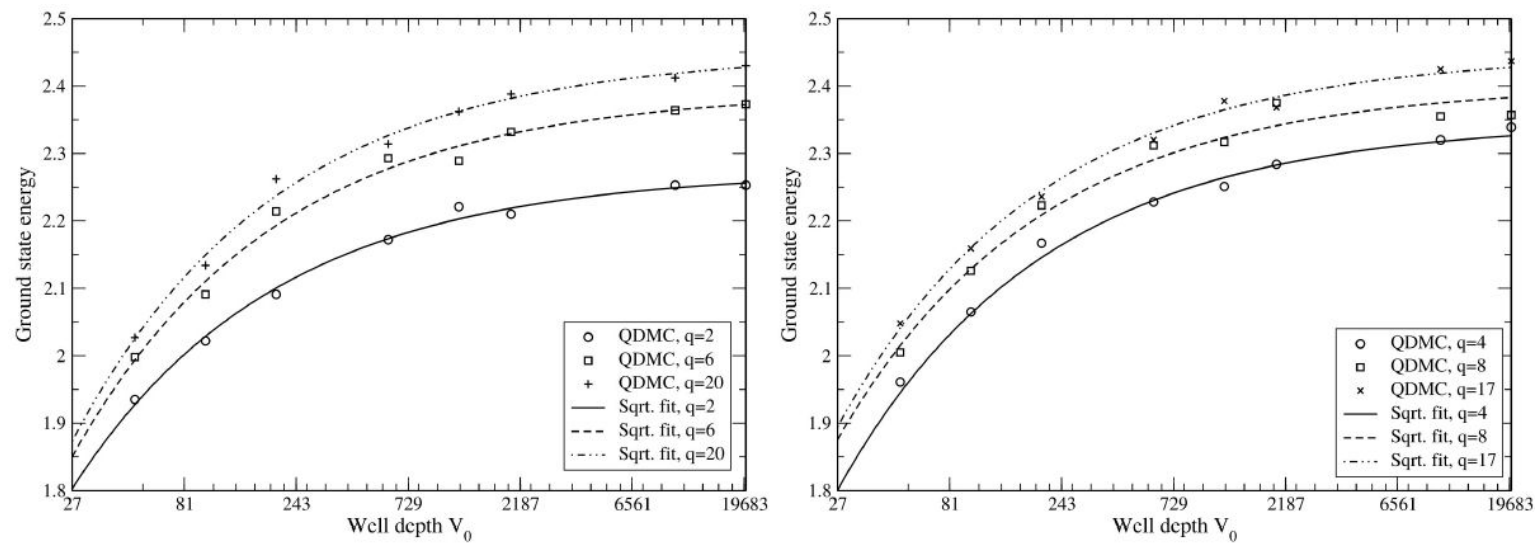

FIGURE 5. QDMC ground state energies of one electron as function of $V_{0}$ for a supercircular potential well of area 4.0, shape given by Eq. (5) and several values of exponent $q$. The square root type curve fits of Eq. (21) are also shown in the figure.
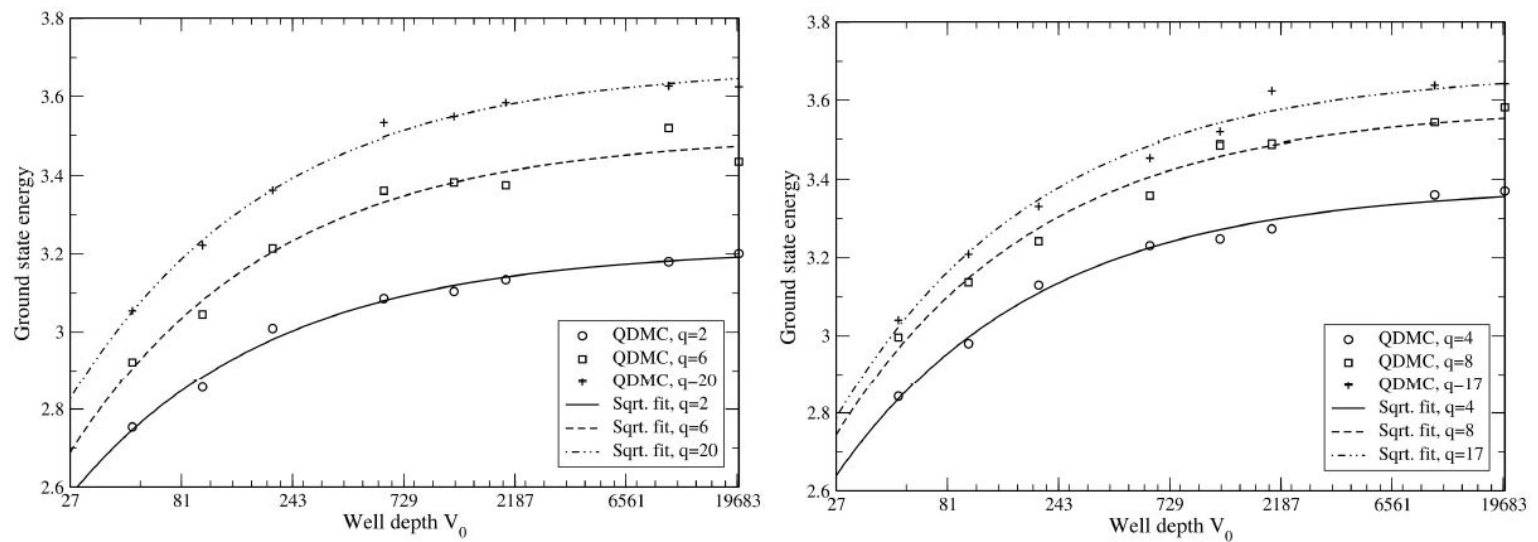

FIGURE 6. QDMC ground state energies of one electron as function of $V_{0}$ for a superspherical potential well of volume 8.0, shape of Eq. (6) and several values of exponent $q$. The square root type curve fits of Eq. (21) are also shown in the figure.

show the data points and curve fits, with $V_{0}$ on the horizontal axis and $E_{0}$ on the vertical.

\subsection{QDMC results for two particles in $2 D$}

The QDMC ground state energies, $E_{0}$, for two interacting or non-interacting electrons in a $2 \mathrm{D}$ potential well of depth $V_{0}=200$ or $V_{0}=20000$ are shown in Fig. 7 with the value of parameter $s$ varying from 1.0 to 4.0. The area of the potential well is $A=\pi$ when $s=1$ regardless of what the value of $q$ is and $A=\pi s^{2}$ for other values of $s$, to make the results easier to compare. For $q=2$ it holds that $s=r$, for $q=8$ the relation is $s \approx 1.1162 r$ and for $q=\infty, s \approx 1.1284 r$ with $r$ as in Eq. 5. More generally, for a given value of $q$ and 2-dimensional space the connection between $s$ and $r$ is $s=\sqrt{\operatorname{Vol}(r, 2, q) / \pi}$ with $\operatorname{Vol}(r, n, q)$ as in Eq. (7).

In the graphs, there is also a curve fit of form $E_{0}(r) \propto$ $r^{-2}$ made for each $q=8$ data point set. The energy eigenvalues of a set of non-interacting particles, in a potential well deep enough for approximation $V_{0}=\infty$ to be valid, are proportional to reciprocal square of the well diameter. From Fig. 7 it is easy to see that this type of function can be quite ac- curately fitted to the $q=8$ data points for noninteracting electrons, even for the lower well depth $V_{0}=200$, but not accurately at all to the point sets with Coulomb potential included.

The expectation values $\left\langle r_{12}\right\rangle$ are shown as function of $s$ for potential wells of both depths in Fig. 7. Parabolas $\left\langle r_{12}\right\rangle(s)=c_{2} s^{2}+c_{1} s+c_{0}$ have also been fitted in each set of data points, and the result is that each point set is close to being on the same line with $\left|c_{1}\right|>50\left|c_{2}\right|$.

The effect of making the parameter $\epsilon$ (meant to prevent division by zero when calculating the electron-electron Coulomb potential) smaller by a factor of 100 was also tested in the $q=2, V_{0}=200$ case with two interacting electrons. Figure 8 shows how much difference this causes in the $E_{0}(s)$ data points. The value $\epsilon=0.001$ may look large, especially when it's inside the square root in $r_{12}=\sqrt{\left(x_{1}-x_{2}\right)^{2}+\left(y_{1}-y_{2}\right)^{2}+\epsilon}$, but the graph in Fig. 8 shows that not much improvement can be made by setting a smaller value.

The Appendix contains the tables of numerical data for $E_{0}$ (Fig. 7) and $\left\langle r_{12}\right\rangle$ (Fig. 8) as a function of $r$ and for different well depths. 

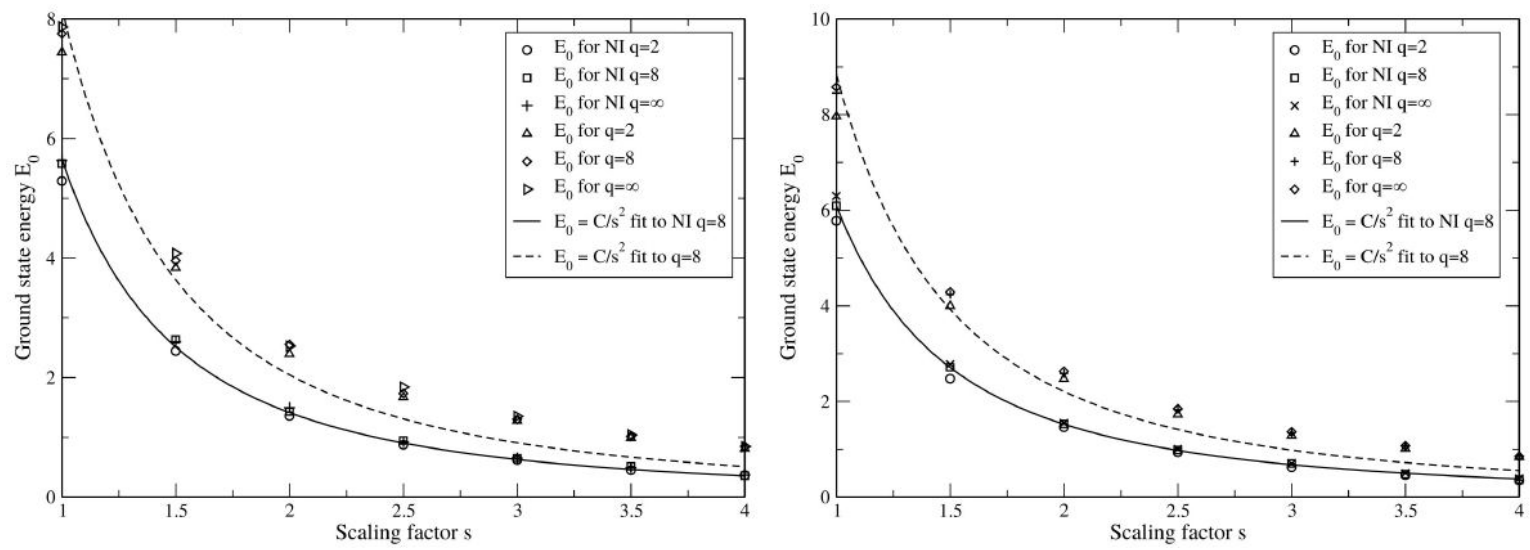

FIGURE 7. QDMC ground state energy $E_{0}$ for two interacting or non-interacting (NI) electrons in a supercircular potential well of depth $V_{0}=200$ (left) or $V_{0}=20000$ (right). The parameter $q$ is the same as in Eq. (5) and $s$ a linear scaling factor, with the area of the supercircle being $\pi s^{2}$ for a given value of $s$. An $E_{0}(s) \propto s^{-2}$ nonlinear fit has been made to the $q=8$ data points to demonstrate the difference caused by the presence of Coulomb interaction.
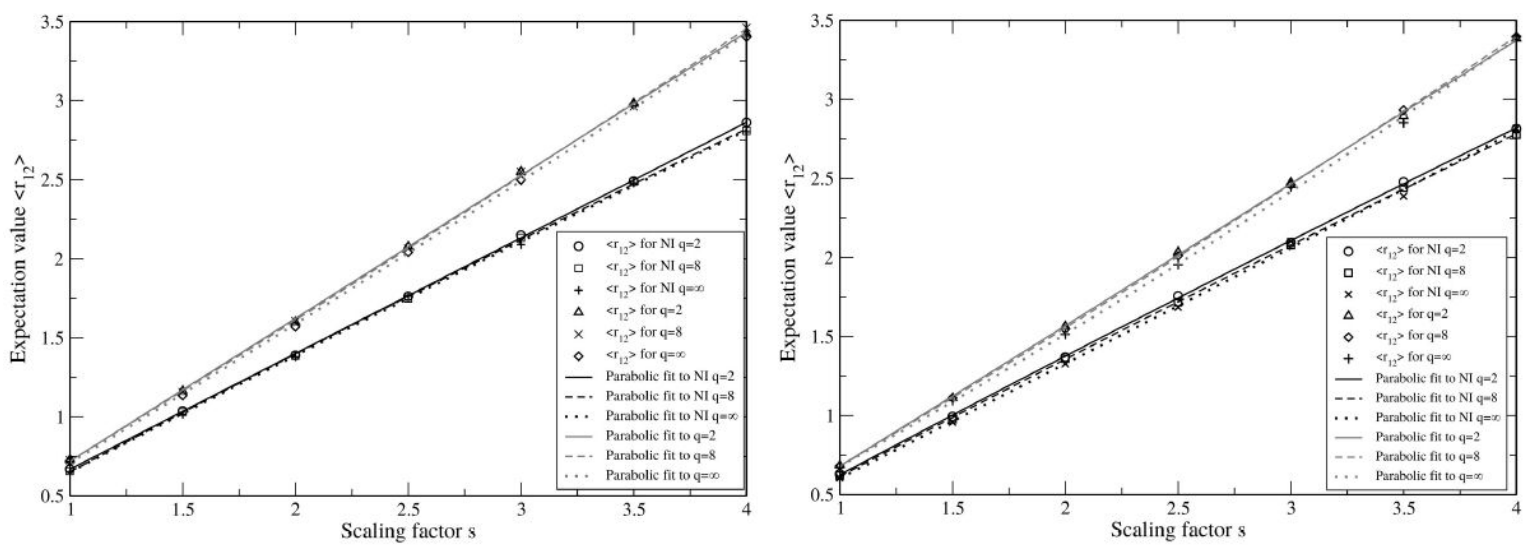

FIGURE 8. QDMC expectation values $\left\langle r_{12}\right\rangle$ for two interacting or non-interacting (NI) electrons in a supercircular potential well of depth $V_{0}=200$ (left) or $V_{0}=20000$ (right), parameter $q$ as in Eq. (5) and $s$ a linear scaling factor such that the area of the supercircle is $\pi s^{2}$ for a given value of $s$.

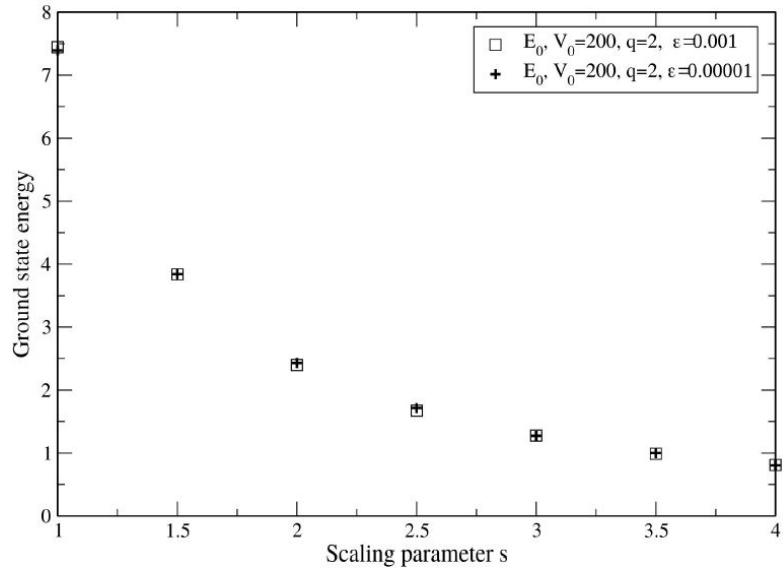

FIGURE 9. The values of $E_{0}(s)$, obtained with QDMC, for two interacting electrons in a potential well with $q=2$ and $V_{0}=200$ and with two different values of cutoff parameter $\epsilon$.

\subsection{Implicit time stepping results for one particle in 2D}

When exponential functions of type (20) were fitted in the $E_{0}$ and $E_{1}$ values obtained with the IFD method and imaginary time propagation, the parameters $A_{0}, A_{1}$ and $A_{2}$ of Eq. (20) were found to be as in Table II. The data points $E_{0}(q)$ and $E_{1}(q)$ are shown with the nonlinear fits in Fig. 10.

TABLE II. The parameters $A_{0}, A_{1}$ and $A_{2}$ of (21), with the nonlinear fit done to $E_{0}(q)$ and $E_{1}(q)$ values obtained with finite difference imaginary time propagation for a single electron in a $2 \mathrm{D}$ potential well of area 4.0, depth $V_{0}$ and shape given by (5).

\begin{tabular}{cccc}
\hline eigenvalue & $A_{0}$ & $A_{1}$ & $A_{2}$ \\
\hline$E_{0}\left(V_{0}=2000\right)$ & 2.3896 & 2.2072 & -0.25035 \\
$E_{1}\left(V_{0}=2000\right)$ & 5.9779 & 5.6029 & -0.22288 \\
$E_{0}\left(V_{0}=20000\right)$ & 2.4366 & 2.2448 & -0.25274 \\
$E_{1}\left(V_{0}=20000\right)$ & 6.0957 & 5.6985 & -0.22526 \\
\hline
\end{tabular}



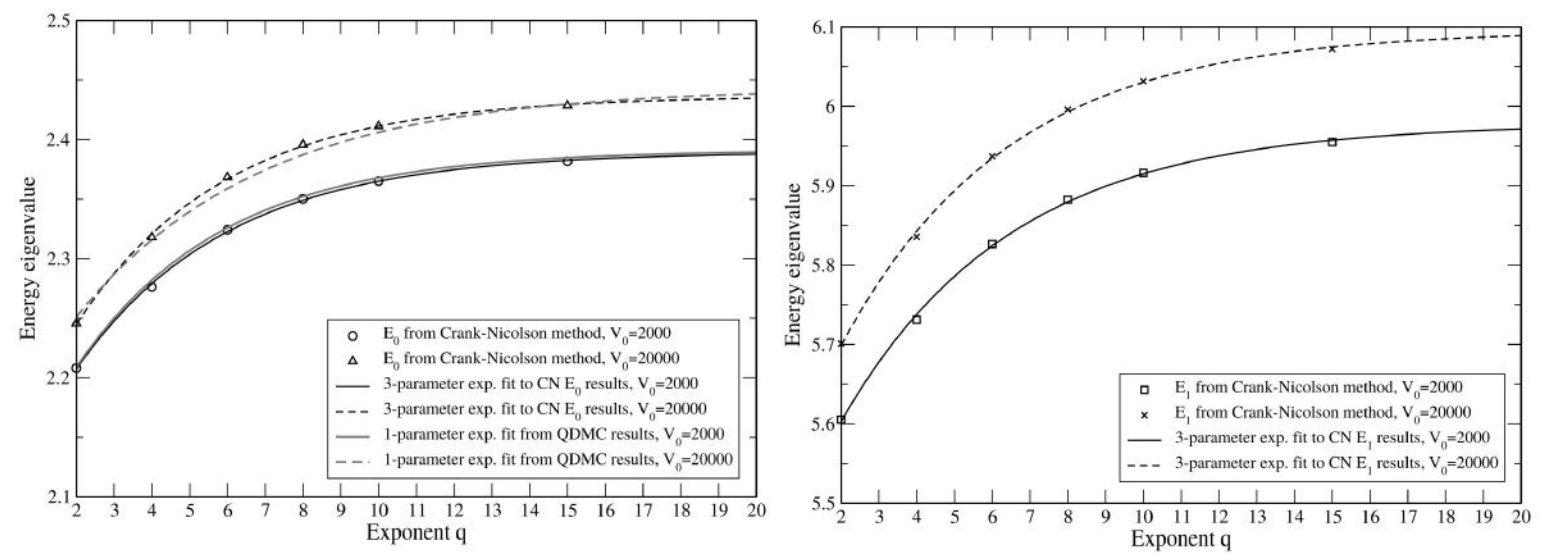

FIGURE 10. Energy eigenvalues $E_{0}$ (left) and $E_{1}$ (right) for one electron in a 2D potential well of shape (5), area 4.0 and depth $V_{0}=2000$ or $V_{0}=20000$. The results were calculated by imaginary time propagation done with implicit finite differencing and operator splitting. In the left image, the equivalent QDMC results are also plotted for comparison.

The $A_{0}$ values (limit of $E$ at $q \rightarrow \infty$ ) for the ground states of $V_{0}=2000$ and $V_{0}=20000$ are 2.3896 and 2.4366 . When these are compared to values in Table I, 2.3912 and 2.4430 , the error in the first case is less than $0.1 \%$ and that in the second, less than $0.3 \%$. The corresponding ground state $A_{1}$ values are 2.2072 and 2.2448 , representing the limit of $E_{0}$ for $q \rightarrow 2$. The equivalent values in Table I, calculated with the shooting method, are 2.2087 and 2.2511. The difference between the IFD and shooting method values is less than $0.3 \%$ in both.

The $A_{0}$ values for the first excited state, calculated with IFD method, were 5.9779 and 6.0957. The 1st excited state energy $E_{1}$ can also be calculated for the $q \rightarrow \infty$ system with Eqs. (12) and (13), and the results for $V_{0}=2000$ and $V_{0}=20000$ are 5.9779 and 6.1073 . The differences between the IFD results and those from (12) and (13) are here less than $0.2 \%$.

The value for the second excited state energy $E_{2}$ from the IFD simulations done for $q=2$ case were 11.6338 for $V_{0}=2000$ and 11.8318 for $V_{0}=20000$. When a shooting method calculation was done for these two systems with a rotation invariant 2nd excited state, the results were 11.635 for $V_{0}=2000$ and 11.861 for $V_{0}=20000$. Here the percentual error is again less than $0.3 \%$. The consistency between $q=2$ and $q=\infty$ results obtained with different methods makes it seem likely that the results of Fig. 10 are close to correct also for values of $q$ between the two extremes.

\section{Conclusion}

In this work, the ground state energies of potential wells of Lamé circle or Lamé sphere shape were calculated for a large number of combinations of well depth $V_{0}$ and the exponent $q$ in Eq. (6). In most calculations the parameter $r$ of (6) was defined as a function of $q, r:=r(q)$, to keep the area or volume of the potential well at a $q$-independent constant value. It was also shown that simple functions of 2 or 3 parameters can be accurately fitted in the $E_{0}\left(q, V_{0}\right)$ data points, but there is no obvious way to show mathematically that the function $E_{0}(q)$ could be written as a sum of an exponential plus small correction terms. Based on the more accurate data points in Fig. 10, it does not even seem completely impossible that the exponential dependence of $E_{0}$ on $q$ with $V_{0}$ and $\mathcal{V}$ kept constant could be the exact solution of the problem.

The most surprising fact in this was that the multiplier $A_{2}$ in the optimal exponential function fit seemed to have a similar value of $-0.30 \leq A_{2} \leq-0.20$ for all well depths, volumes and both in $2 \mathrm{D}$ and $3 \mathrm{D}$. Furthermore, most of the variation in the value of that parameter appeared to be random numerical error, because the value didn't consistently change to either direction when the well depth or volume was altered. The variation in those values was greater for the $2 \mathrm{D}$ potential wells for which the calculations were done with a smaller number of random walkers. A simple back-of-theenvelope way to approximate the $E_{0}$ for some $V_{0}, \mathcal{V}$ and $q$ could be to set the $A_{0}$ and $A_{1}$ of (20) to the $E_{0}(q=\infty)$ and $E_{0}(q=2)$ values obtained from the Eqs. (12) to (16) and guess that $A_{2} \approx-0.24$.

In the case of $2 \mathrm{D}$ potential wells, calculations were also made for the first excited state energy $E_{1}$ of one electron, and the ground state energy $E_{0}$ and expected electron-electron distance $\left\langle r_{12}\right\rangle$ of two electrons confined in a 2D Lamé circle. The value of $\left\langle r_{12}\right\rangle$ was found to increase almost linearly as a function of a scaling parameter $s$.

In the cases where it was possible to compare results calculated by different means for the same system, there was a good agreement between QDMC, IFD imaginary time propagation, shooting method and graphical solution results. Especially the consistency of the QDMC and more accurate IFD results in the left image of Fig. 10 is a good sign that the 2D QDMC results are correct enough despite the significant random error in individual data points (Fig. 2).

The results are an extension of those in Ref. [10], where only $2 \mathrm{D}$ potential wells of infinite depth were considered. As 
remarked in that publication, this kind of results can be used for estimating the cube- or spherelikeness of a real physical potential well system, such as an electron in a quantum dot or a vacant site of a crystal lattice. Another possible application of the content of this article could be as an example problem for students of computational chemistry and physics.

One problem related to the material in this article is the behavior of two oppositely charged particles in a $2 \mathrm{D}$ or $3 \mathrm{D}$ potential well. This could be described as a hydrogen atom [21, 22] or positronium [23] being compressed by an external force, and the interparticle distance $\left\langle r_{12}\right\rangle$ would approach some finite limiting value with increasing scaling parameter $s$.

It has probably been known since the early years of quantum mechanics that a system of a particle interacting with a 3D spherical potential well doesn't necessarily have even one bound state, while one in a rectangular potential well always does. The existence of a bound state in the spherical well depends on whether its $V_{0}$ and radius are large enough. One unanswered question is whether there exists some finite minimum value of the exponent $q$ in Eq. (6) that guarantees the existence a bound state for a potential well with that bounding surface. One possible way to heuristically probe the situation would be to add more data points of low $V_{0}$ to the data in Figs. 5 and 6 and extrapolate to $V_{0} \rightarrow 0$, but an actual mathematical proof would be a completely different task.

\section{Appendix A}

This Appendix contains tables of numerical data related to the results presented in the main text. Tables III and IV contain the values of parameters $A_{0}, A_{1}$ and $A_{2}$ obtained when fitting the exponential function of Eq. (20) to the data points of $E_{0}$ as function of $q$ with $V_{0}$ and $A=4.0$ or $\mathcal{V}=8.0 \mathrm{kept}$ constant. The parameters were found with the "Non-linear curve fitting" functionality of the Linux application Grace5.1.25, using at least 15 iterations. The 1-parameter fit was done with $A_{0}$ and $A_{1}$ fixed to the values $E_{0}(q=\infty)$ and $E_{0}(q=2)$ calculated with the non-algebraic equations of Subsec. 2.1, and the 3-parameter fit with all parameters being variable. This data is for the systems with only one electron

TABLE III. The numbers $A_{0}, A_{1}$ and $A_{2}$ of Eq. (20) obtained in the three- (left) and one-parameter (right) fits to the data points $E_{0}(q)$ of one particle in a 2D Lamé circle potential well of area 4.0.

\begin{tabular}{cccc|cc}
\hline$V_{0}$ & $A_{0}$ & $A_{1}$ & $A_{2}$ & $V_{0}$ & $A_{2}$ \\
\hline 50 & 2.0353 & 1.9259 & -0.2172 & 50 & -0.2322 \\
100 & 2.1553 & 2.0077 & -0.2136 & 100 & -0.2206 \\
200 & 2.2387 & 2.0894 & -0.3030 & 200 & -0.3252 \\
600 & 2.3239 & 2.1613 & -0.3209 & 600 & -0.2979 \\
1200 & 2.3783 & 2.2010 & -0.1680 & 1200 & -0.1989 \\
2000 & 2.3978 & 2.2070 & -0.2398 & 2000 & -0.2567 \\
10000 & 2.4259 & 2.2533 & -0.2438 & 10000 & -0.2385 \\
20000 & 2.4428 & 2.2613 & -0.1931 & 20000 & -0.2059 \\
\hline
\end{tabular}

TABLE IV. The numbers $A_{0}, A_{1}$ and $A_{2}$ of Eq. (20) obtained in the three- (left) and one-parameter (right) fits to the data points $E_{0}(q)$ of one particle in a 3D Lamé sphere potential well of volume 8.0.

\begin{tabular}{cccc|cc}
\hline$V_{0}$ & $A_{0}$ & $A_{1}$ & $A_{2}$ & $V_{0}$ & $A_{2}$ \\
\hline 50 & 3.0568 & 2.7303 & -0.2341 & 50 & -0.2248 \\
100 & 3.2389 & 2.8494 & -0.2320 & 100 & -0.2344 \\
200 & 3.3644 & 2.9857 & -0.2205 & 200 & -0.2478 \\
600 & 3.5278 & 3.0599 & -0.2060 & 600 & -0.2386 \\
1200 & 3.5523 & 3.0950 & -0.2394 & 1200 & -0.2324 \\
2000 & 3.6118 & 3.1186 & -0.1973 & 2000 & -0.2171 \\
10000 & 3.6214 & 3.1539 & -0.2866 & 10000 & -0.2393 \\
20000 & 3.6582 & 3.1786 & -0.2545 & 20000 & -0.2464 \\
\hline
\end{tabular}

TABLE V. The numbers $A_{0}, A_{1}$ and $A_{2}$ of Eq. (20) obtained in the three-parameter exponential fit to the data points $E_{0}(q)$ of one particle in a 3D Lamé sphere potential well of smaller volume 2.0.

\begin{tabular}{cccc}
\hline$V_{0}$ & $A_{0}$ & $A_{1}$ & $A_{2}$ \\
\hline 600 & 8.5132 & 7.4299 & -0.2790 \\
2000 & 8.8176 & 7.7465 & -0.2459 \\
20000 & 9.0608 & 7.8903 & -0.2851 \\
\hline
\end{tabular}

TABLE VI. The parameters $A_{0}$ and $A_{1}$ of Eq. (21), with the nonlinear fit done to QDMC $E_{0}$ data points of one electron in a 2D (left) or 3D (right) potential well of shape (5) or (6), area 4.0 (2D) or volume $8.0(3 \mathrm{D})$.

\begin{tabular}{cccccc}
\hline$q$ & $A_{0}$ & $A_{1}$ & $q$ & $A_{0}$ & $A_{1}$ \\
\hline 2 & 2.2735 & 2.4469 & 2 & 3.2149 & 3.3093 \\
4 & 2.3461 & 2.8354 & 4 & 3.3835 & 3.8694 \\
6 & 2.3924 & 2.8191 & 6 & 3.5055 & 4.2434 \\
8 & 2.4025 & 2.7389 & 8 & 3.5850 & 4.3722 \\
10 & 2.4350 & 3.0404 & 10 & 3.5948 & 4.0891 \\
12 & 2.4516 & 3.1079 & 12 & 3.6685 & 4.5119 \\
17 & 2.4478 & 2.8764 & 17 & 3.6755 & 4.6182 \\
20 & 2.4487 & 2.9921 & 20 & 3.6769 & 4.4172 \\
\hline
\end{tabular}

in the $2 \mathrm{D}$ or $3 \mathrm{D}$ potential well, and corresponds to the results of Figs. 2 and 3. The Table $\mathrm{V}$ contains the parameters $A_{0}$, $A_{1}$ and $A_{2}$ for the $V_{0}=600, V_{0}=2000$ and $V_{0}=20000$ $3 \mathrm{D}$ potential well of smaller volume $\mathcal{V}=2.0$, with all three parameters being free (Fig. 4).

The Table VI contains the parameters $A_{0}$ and $A_{1}$ from the fitting of the square root type function $(21)$ to the data with $E_{0}$ values as function of $V_{0}$ and with $q$ and $\mathcal{V}$ kept constant. The graphs of these functions and the associated data points are shown in the Figs. 5 and 6 of the main article.

The Tables VII and VIII contain the $E_{0}$ values of a system of two electrons in a potential well of depth $V_{0}=2000$ or $V_{0}=20000$, with the parameter $s$ (and the surface area of the 2D well) being variable. Parameter $s$ is defined as $s=\sqrt{\mathcal{V} / \pi}$, 
TABLE VII. The ground state energy of two interacting or noninteracting electrons in a $2 \mathrm{D}$ potential well of depth $V_{0}=200$ and shape given by Eq. (5) with $q=2,8$ or $\infty$ and $r$ defined as function of $s$ so that the area of the well is $\pi s^{2}$.

\begin{tabular}{cccccccc}
\hline \multirow{2}{*}{$s$} & \multicolumn{3}{c}{ Non-interacting $E_{0}$} & & \multicolumn{3}{c}{ Interacting $E_{0}$} \\
\cline { 2 - 3 } \cline { 6 - 8 } & $q=2$ & $q=8$ & $q=\infty$ & & $q=2$ & $q=8$ & $q=\infty$ \\
\hline 1.0 & 5.289 & 5.581 & 5.630 & & 7.440 & 7.750 & 7.864 \\
1.5 & 2.444 & 2.639 & 2.597 & & 3.837 & 3.954 & 4.079 \\
2.0 & 1.358 & 1.429 & 1.496 & & 2.395 & 2.553 & 2.525 \\
2.5 & 0.875 & 0.945 & 0.924 & & 1.671 & 1.734 & 1.839 \\
3.0 & 0.621 & 0.650 & 0.654 & & 1.279 & 1.301 & 1.350 \\
3.5 & 0.449 & 0.515 & 0.494 & & 0.990 & 1.016 & 1.040 \\
4.0 & 0.366 & 0.357 & 0.368 & & 0.812 & 0.845 & 0.845 \\
\hline
\end{tabular}

TABLE VIII. The ground state energy of two interacting or noninteracting electrons in a 2D potential well of depth $V_{0}=20000$ and shape given by Eq. (5) with $q=2,8$ or $\infty$ and $r$ defined as function of $s$ so that the area of the well is $\pi s^{2}$.

\begin{tabular}{cccccccc}
\hline \multirow{2}{*}{$s$} & \multicolumn{3}{c}{ Non-interacting $E_{0}$} & & \multicolumn{3}{c}{ Interacting $E_{0}$} \\
\cline { 2 - 3 } \cline { 6 - 8 } \cline { 6 - 8 } & $q=2$ & $q=8$ & $q=\infty$ & & $q=2$ & $q=8$ & $q=\infty$ \\
\hline 1.0 & 5.780 & 6.093 & 6.300 & & 7.963 & 8.450 & 8.576 \\
1.5 & 2.475 & 2.717 & 2.778 & & 3.998 & 4.238 & 4.289 \\
2.0 & 1.462 & 1.529 & 1.548 & & 2.472 & 2.585 & 2.628 \\
2.5 & 0.935 & 0.983 & 1.006 & & 1.727 & 1.831 & 1.846 \\
3.0 & 0.623 & 0.703 & 0.702 & & 1.286 & 1.342 & 1.363 \\
3.5 & 0.458 & 0.480 & 0.497 & & 1.013 & 1.066 & 1.072 \\
4.0 & 0.348 & 0.361 & 0.388 & & 0.836 & 0.847 & 0.848 \\
\hline
\end{tabular}

TABLE XIX. The electron-electron distance expectation value $\left\langle r_{12}\right\rangle$ of two interacting or non-interacting electrons in a $2 \mathrm{D}$ potential well of depth $V_{0}=200$ and shape given by Eq. (5) with $q=2,8$ or $\infty$ and scaling factor $s$ as in first column.

\begin{tabular}{cccccccc}
\hline \multirow{2}{*}{$s$} & \multicolumn{3}{c}{ Non-interacting $\left\langle r_{12}\right\rangle$} & & \multicolumn{3}{c}{ Interacting $\left\langle r_{12}\right\rangle$} \\
\cline { 2 - 3 } \cline { 7 - 8 } \cline { 6 - 7 } & $q=2$ & $q=8$ & $q=\infty$ & & $q=2$ & $q=8$ & $q=\infty$ \\
\hline 1.0 & 0.6697 & 0.6561 & 0.6540 & & 0.7391 & 0.7262 & 0.7205 \\
1.5 & 1.0381 & 1.0310 & 1.0155 & & 1.1607 & 1.1571 & 1.1352 \\
2.0 & 1.3901 & 1.3862 & 1.3800 & & 1.5883 & 1.6066 & 1.5700 \\
2.5 & 1.7640 & 1.7498 & 1.7674 & & 2.0551 & 2.0651 & 2.0417 \\
3.0 & 2.1498 & 2.1329 & 2.0900 & & 2.5454 & 2.5502 & 2.4979 \\
3.5 & 2.4908 & 2.4883 & 2.4797 & & 2.9949 & 2.9614 & 2.9740 \\
4.0 & 2.8618 & 2.8084 & 2.8041 & & 3.4793 & 3.4610 & 3.4022 \\
\hline
\end{tabular}

TABLE X. The electron-electron distance expectation value $\left\langle r_{12}\right\rangle$ of two interacting or non-interacting electrons in a $2 \mathrm{D}$ potential well of depth $V_{0}=20000$ and shape given by Eq. (5) with $q=2$, 8 or $\infty$ and scaling factor $s$ as in first column.

\begin{tabular}{cccccccc}
\hline \multirow{2}{*}{$s$} & \multicolumn{3}{c}{ Non-interacting $\left\langle r_{12}\right\rangle$} & & \multicolumn{3}{c}{ Interacting $\left\langle r_{12}\right\rangle$} \\
\cline { 2 - 4 } \cline { 7 - 8 } & $q=2$ & $q=8$ & $q=\infty$ & & $q=2$ & $q=8$ & $q=\infty$ \\
\hline 1.0 & 0.6310 & 0.6209 & 0.6067 & & 0.6854 & 0.6815 & 0.6728 \\
1.5 & 0.9948 & 0.9776 & 0.9577 & & 1.1115 & 1.1125 & 1.0952 \\
2.0 & 1.3705 & 1.3608 & 1.3280 & & 1.5655 & 1.5470 & 1.5145 \\
2.5 & 1.7580 & 1.7177 & 1.6865 & & 2.0356 & 2.0113 & 1.9551 \\
3.0 & 2.0952 & 2.0799 & 2.1025 & & 2.4730 & 2.4597 & 2.4444 \\
3.5 & 2.4779 & 2.4407 & 2.3883 & & 2.8936 & 2.9318 & 2.8509 \\
4.0 & 2.8133 & 2.7750 & 2.8167 & & 3.3847 & 3.3959 & 3.3985 \\
\hline
\end{tabular}

$s=\sqrt{\mathcal{V} / \pi}$, with $\mathcal{V}$ the area of the well. The same letter $\mathcal{V}$ as for the volume of a 3D potential well is used here because surface area is just its 2-dimensional equivalent. The data of Tables VII and VIII is the same as used for producing Fig. 7 of the article.

The expectation values of the electron-electron distance, in the ground state of two electrons in the 2D potential well, are shown as a function of $s$ in Tables IX and X. Figure 8 of the main article contains the same results. The quantity $\left\langle r_{12}\right\rangle$ increases almost linearly as a function of $s$, but this property would probably not hold for small radii $s<0.5$ when the confining force is not enough to keep both electrons inside the potential well.

\section{Acknowledgements}

The author wants to thank the editor and referees for prompt review process of this article. 
1. P. Tiwald et al., Ab initio perspective on the Mollwo-Ivey relation for F centers in alkali halides, Phys. Rev. B 92 (2015) 144107, https://doi.org/10.1103/PhysRevB.92.144107

2. A. Popov, E. Kotomin and J. Maier, Basic properties of the F-type centers in halides, oxides and perovskites, Nucl. Instrum. Methods Phys. Res. B 268 (2010) 3084, https://doi.org/10.1016/j.nimb.2010.05.053.

3. R. Li, Z. Liu, Y. Wu and C. S. Liu, The impacts of the quantumdot confining potential on the spin-orbit effect, Sci. Rep. 8 (2018) 7400, https://doi.org/10.1038/s41598-018-25692-2

4. K. L. Jahan, B. Boyacioglu and A. Chatterjee, Effect of confinement potential shape on the electronic, thermodynamic, magnetic and transport properties of a GaAs quantum dot at finite temperature, Sci. Rep. 9 (2019) 15824, https://doi.org/10.1038/s41598-019-52190-w

5. J. Autschbach, Why the Particle-in-a-Box Model Works Well for Cyanine Dyes but Not for Conjugated Polyenes, J. Chem. Educ. 84 (2007) 1840, https://doi.org/10.1021/ed084p1840

6. M. Mauksch and S. B. Tsogoeva, Spin-paired solvated electron couples in alkali-ammonia systems, Phys. Chem. Chem. Phys. 20 (2018) 27740, https://doi.org/10.1039/C8CP05058A

7. H. J. Maris, Electrons in liquid helium, J. Phys. Soc. Jpn. 77 (2008) 111008, https://doi.org/10.1143/JPSJ.77.111008

8. Y. Xing and H. J. Maris, Electrons and Exotic Ions in Superfluid Helium-4, J. Low Temp. Phys. 201 (2020) 634, https://doi.org/10.1007/s10909-020-02422-5

9. A. Slavik and D. Sulc, Maximal volumes of $n$-dimensional balls in the p-norm, Arch. Math. 114 (2020) 305, https://doi.org/10.1007/s00013-019-01394-7

10. N. Bera, J.K. Bhattacharjee, S. Mitra, and S. P. Khastgir, Energy levels of a particle confined in a super-circular box, Eur. Phys. J. D 46 (2008) 41, https://doi.org/10.1140/epjd/e2007-00282-6

11. J. Gielis, The Geometrical Beauty of Plants (Atlantic press, 2017) pp. 53-55.

12. S. Onaka, Simple equations giving shapes of various convex polyhedra: the regular polyhedra and polyhedra composed of crystallographically low-index planes, Philos. Mag. Lett. 86 (2009) 175, https://doi.org/10.1080/09500830600603050

13. A. P. Zhou and W. D. Sheng, Electron and hole effective masses in self-assembled quantum dots, Eur. Phys. J. B 68 (2009) 233, https://doi.org/10.1140/epjb/e2009-00098-2

14. N. Vukmirović, L-W. Wang, Quantum Dots: Theory, Comprehensive Nanoscience and Technology 1 (2011) 189, https://doi.org/10.1016/B978-0-12-374396-1.00027-1

15. P. S. Drouvelis, P. Schmelcher and F. K. Diakonos, Probing the shape of quantum dots with magnetic fields, Phys. Rev. B 69 (2004) 155312, https://doi.org/10.1103/PhysRevB.69.155312

16. I. Kosztin, B. Faber and K. Schulten, Introduction to the Diffusion Monte Carlo Method, Am. J. Phys. 64 (1996) 633, https://doi.org/10.1119/1.18168

17. B. H. Bransden and C. J. Joachain, Quantum Mechanics 2nd Ed. (Pearson Education Limited, 2000).

18. O. Bolina, Trotter formula and thermodynamic limits, 2002, arXiv:physics/0202003

19. W. H. Press, S. A. Teukolsky, W. T. Vetterling and B. P. Flannery, Numerical Recipes: The Art of Scientific Computing 3rd Ed. (Cambridge University Press, 2007).

20. Z. Sun, W. Yang and D. H. Zhang, Higher-order split operator schemes for solving the Schrödinger equation in the timedependent wave packet method: applications to triatomic reactive scattering calculations, Phys. Chem. Chem. Phys. 14 (2012) 1827, https://doi.org/10.1039/C1CP22790D

21. K. D. Sen, Electronic Structure of Quantum Confined Atoms and Molecules, (Springer International Publishing Switzerland, 2014), pp. 59-89.

22. N. Aquino and E. Castaño, The confined two-dimensional hydrogen atom in the linear variational approach, Rev. Mex. Fis. E 51 (2005) 126.

23. P. Hautojärvi, M. T. Loponen, and K. Rytsölä, Positronium bubble in liquid helium, J. Phys. B: Atom. Molec. Phys. 9 (1976) 411, https://doi.org/10.1088/0022-3700/9/3/010 